\title{
Modelo de Política Pública de I+D+i, Para Liderar y Coordinar el Sistema Regional de Innovación en Apoyo a las PYMIS. Caso de Estudio del Estado de Bolívar,Venezuela.
}

\author{
Public Policy Model for R\&D\&I to Lead and Coordinate the Regional \\ Innovation System in Support of SMls. Case Study of Bolivar State, Venezuela
}

\author{
Alexander Piñero', Carlos Rodríguez Monroy², Minerva Arzola ${ }^{3}$
}

\begin{abstract}
El presente proyecto planteó como objetivo diseñar un Modelo de Política Pública de I+D+i para liderar, coordinar y direccionar el sistema regional de innovación en apoyo al sector de las PYMIS, caso de estudio el estado Bolívar, Venezuela. El proyecto representa una investigación no experimental de tipo proyectivo y se divide en dos partes. Primero se diagnóstica, se evalúa y se describe la situación actual del SRI y su vinculación con las PYMIS. En la segunda parte se diseña y se propone un modelo de política pública de I+D+i para fortalecer la capacidad de innovación en las PYMIS.
\end{abstract}

Keywords: política publica de i+d+i; sistema regional de innovación; capacidad de innovación; pymis.

This proposed project aims to design a Model of Public Policy for R\&D \&l to lead, coordinate and direct the Regional Innovation System (RIS) in support of the SMI sector. The case study of the Bolivar State, Venezuela, is analyzed. The project represents a no- experimental research of projective type and is divided into two parts. In the first one, the current state of the SRI and its relationship with the SMIs is diagnosed, evaluated and described. In the second part, a model of public policy for R\&D\&l is designed and proposed in order to strengthen the capacity for innovation in SMls.

Keywords: public policy r\&d\&i; regional innovation system (ris); innovation capacity; small and medium industries (smis).

\footnotetext{
'UNEG Coordinación de Postgrado Urbanización Chilemex. Código postal 8050. Phone: 0058-416 3904818.

E-mail: alexanderpinerol2@gmail.com.

${ }^{2}$ Dpto. de Organización, Administración de Empresas y Estadística. Universidad Politécnica de Madrid (UPM), José Gutiérrez Abascal, 2. 28006 Madrid, España. Phone: 0034 - 913364265. E-mail: crmonroy@etsii.upm.es.

${ }^{3}$ UNEXPO Coordinación de Postgrado Urbanización Villa Asia. Código postal 8050. Phone: 0058-4I 29497380.

E-mail: minervarzola@yahoo.com.
} 


\section{Introducción}

La generación de conocimientos para la innovación tecnológica está permitiendo a la administración gubernamental, reconocer la importancia de desarrollar las actividades de Investigación, Desarrollo Tecnológico e Innovación(l+D+i) para mejorar la competitividad del sector industrial, convirtiéndose como un factor determinante para las empresas y para las políticas públicas de I+D+i, de entender que la innovación es el motor no sólo para el crecimiento, fortalecimiento y diversificación de las empresas, sino también en el desarrollo económico y mejora de los indicadores sociales, impactando favorablemente en los beneficios para la población.

El Libro Verde de Innovación de la Comisión Europea (1995) considera la innovación como sinónimo de producir, asimilar y explotar con éxito una novedad, en las esferas económica y social, de forma que aporte soluciones inéditas a los problemas y permita así responder a las necesidades de las personas y de la sociedad.

La Fundación COTEC para la innovación tecnológica en España (2005) describe la innovación como el proceso que convierte conocimiento en Producto Interior Bruto (PIB) y bienestar mediante la creación de nuevos productos o servicios o la mejora de los existentes.

En el Manual de OSLO-OCDE (2006) menciona que el vínculo entre la innovación y el progreso económico es del máximo interés. Es por medio de la innovación que se crea y se difunde un nuevo conocimiento, lo que aumenta el potencial de la economía para desarrollar nuevos productos y métodos de funcionamiento más productivos.

En el Informe elaborado por la Fundación Cotec (2010) sobre la Tecnología e Innovación en España, considera que "la capacidad de innovación tecnológica de un país se apoya fundamentalmente en su esfuerzo de inversión en investigación y desarrollo tecnológico $(I+D)$, en su esfuerzo para conseguir un capital humano capacitado para adquirir conocimientos y desarrollar tecnologías de cualquiera de las formas existentes y en la existencia de un tejido empresarial, que sea capaz de aprovechar las fuentes de conocimiento y tecnología a su alcance para producir productos y servicios novedosos que generen negocio $y$, por tanto, que mejoren su competitividad. Estas premisas son válidas tanto en períodos de bonanza económica como en etapas de crisis, constituyendo la capacidad de innovación uno de los recursos que permiten afrontar mejor estas últimas".

Las condiciones económicas y beneficios de la innovación en las industrias y en la sociedad, serán más favorables en la medida que se diseñen política pública de I+D+i que logren articular e integrar los actores que forman parte del Sistema Innovación (SI), como los son las universidades con sus respectivos centros y laboratorios de Investigación y Desarrollo y su rol de formación de capital humano, las industrias que aplican los conocimientos y agregan valor, la administración pública que definen y coordina Políticas Industriales y de Innovación, entidades financieras de los sectores públicos y privados, y otros organismos asociados al SI.

En regiones donde la cultura y el riesgo a la innovación es muy débil, y con poco nivel de cooperación para la iniciación de proyectos de I+D+i aplicados a la industria, es de vital importancia el rol gubernamental para la coordinación y aplicación de política de I+D+i, adaptadas a los requerimientos de las Pequeñas y Medianas Industrias (PYMIS), garantizando el desarrollo de la I+D para nuevos productos que satisfagan las necesidades del mercado.

En el otro caso, donde la cultura de la cooperación en I+D+i se encuentra en niveles de avanzadas, y con aprendizaje continuo en las industrias, la coordinación gubernamental servirá de apoyo de acuerdo a las negociaciones y necesidades presentadas por la relación universidad-industria en los proyectos de I+D+i. Es decir la industria considera las alianzas con los centros de I+D, con las universidades, con las instituciones gubernamentales y otros actores del $\mathrm{SI}$, dentro de las estrategias de innovación como una prioridad para alcanzar nuevos mercados.

En este mismo contexto, González (2008) considera necesario diferenciar entre dos tipos de empresas en la región, las que poseen experiencias en el proceso de innovación y mejores condiciones para actuar en el SRI, y el segundo tipo de empresas reúne a aquellas que no son conscientes de la importancia de la innovación, y presentan debilidades para iniciar por si solas las actividades en materia de I+D+i. Estas empresas requieren del apoyo de política pública de I+D+i, $y$ de las instituciones del SRI.

A finales del 2010, fueron publicados las directrices para las estrategias de la Unión Europea en I+D+i, para el 2020. El principal objetivo para las políticas públicas de $I+D+i$ que define la Comisión Europea (2010a), es la de dedicar el $3 \%$ del PIB de los países de UE-27 en I+D+i. Además establece entre otros objetivos lo relacionado con el desarrollo de las capacidades de investigación e innovación en toda Europa de una manera más cooperativa y coordinada. Recomendando facilitar las condiciones de financiamiento para las PYMIS, promoviendo mayor su participación en los programas de I+D.

En Venezuela a partir del año 1999, la Ciencia, la Tecnología e Innovación (CTI), adquieren por primera vez rango de carácter constitucional, concretamente, en el artículo II0 
de la Republica Bolivariana de Venezuela de 1999. Estable el marco legal que orienta la Política de CTI a nivel nacional. Específicamente el articulo II 0 describe "El Estado reconocerá el interés público de la ciencia, la tecnología, el conocimiento y la innovación y sus aplicaciones y los servicios de información necesarios por ser instrumentos fundamentales para el desarrollo económico, social y político del país, así como para la seguridad y soberanía nacional. Para el fomento de esas actividades, el Estado destinará recursos suficientes y creará el sistema nacional de ciencia y tecnología de acuerdo con la ley. El sector privado deberá aportar recursos para los mismos. El Estado garantizará el cumplimiento de los principios éticos y legales que deben regir las actividades de investigación científica, humanística y tecnológica. La Ley determinará los modos y medios para dar cumplimiento a esta garantía."

El sector de las PYMIS, se encuentra incorporado como uno de los actores del Sistema Nacional de Ciencia, Tecnología e Innovación en Venezuela, definido en Ley Orgánica de Ciencia, Tecnología e Innovación (20I0), en el Plan Nacional de CTI (2005-2030), en el Plan Regional de CTI (2008-2013) del estado Bolívar, y en el Plan de Desarrollo Económico y Social de la Nación (2007-20I3). En la ley para la Promoción y Desarrollo de la Pequeña y Mediana Industria y Demás Unidades de Producción Social del año 2008, y en los planes mencionados, se definen los siguientes objetivos y beneficios a lograr para las PYMIS, representando un importante sector de la economía: La conformación de un sistema de innovación, el estimulo a una mayor inversión en I+D+i, la formación de investigadores, la incorporación de las nuevas tecnologías para el aumento de la productividad, el mejoramiento del sector financiero al servicio de las PYMIS, la formación de redes empresariales, la vinculación con el sector universitario y de investigación, y la transferencia de los resultados de la $I+D+i$ al sector productivo. Con el propósito de incorporar nuevos productos al mercado nacional, que permitan la diversificación de la economía, la sustitución de importaciones y la generación de empleos.

En la LOCTI (20I0), se establece que el Estado Venezolano formulará a través de la autoridad nacional con competencia en materia de ciencia, tecnología, innovación y sus aplicaciones, enmarcado en el Plan Nacional de Desarrollo Económico y Social de la Nación, las políticas públicas dirigidas a la solución de problemas concretos de la sociedad, por medio de la articulación e integración de los sujetos que realizan actividades de ciencia, tecnología, innovación y sus aplicaciones. Además menciona la importancia de diseñar los mecanismos de integración de los actores del Sistema Nacional de Ciencia, Tecnología e Innovación, asignándole la responsabilidad de la coordinación a la autoridad nacional con competencia en materia de CTI.
El estado Bolívar con una superficie de $238.000 \mathrm{Kms} 2$ se encuentra ubicado al sur-este de Venezuela y su capital es ciudad Bolívar. Ocupa el $26,24 \%$ de la superficie del territorio nacional. Ciudad Guayana es la principal región del desarrollo económico del estado, es sede de las empresas Básicas de los sectores siderúrgicos y del aluminio que se encargan desde la extracción, procesamiento y transformación del mineral hierro, y la transformación de la Bauxita en aluminio primario. Además cuenta con el gran potencial hidroeléctrico, garantizando el suministro de energía eléctrica para el funcionamiento de las empresas básicas, para el parque industrial de la región, así como para el desarrollo económico y social del país.

Con relación al sector de la industria del mineral de hierro y del aluminio, las empresas destinan más del 60 por ciento de su producción al mercado internacional, el restante es para el consumo de la industria nacional. A pesar de que el sector de las Pequeñas y Medianas Industrias del estado Bolívar, cuenta con la proximidad a la materia prima, y a las demandas y oportunidades del mercado regional y nacional, no se han desarrollado integralmente en materia de I+D+i, para aprovechar las ventajas comparativas y diseñar nuevos productos con alto contenido de valor agregado regional. Sin embargo, Piñero, Arzola y Rodríguez-Monroy (2008) mencionan que las PYMIS del estado Bolívar, en los últimos años han visto afectada su competitividad por presentar deficiencia en la falta de personal calificado, la escasa vinculación con instituciones de investigación, la baja inversión I+D+i, la ausencia de la aplicación de política de innovación y la desarticulación del sistema regional de innovación, constituyen los principales obstáculos para generar bienes y servicios con un alto valor agregado. Esta situación desequilibra y convierte ineficiente el funcionamiento del SRI del estado Bolívar, e incidiendo en la baja capacidad de la I+D+i en las PYMIS.

En la elaboración del Plan Regional de Ciencia, Tecnología e Innovación (2008) del estado Bolívar, se diagnosticaron las problemáticas que afectan la capacidad innovadora del sector de las PYMIS. Los siguientes son los puntos críticos que deben afrontar las políticas públicas de CTI: La carencia de una sólida plataforma tecnológica e industrial que permita aprovechar los recursos minerales y materiales existentes en la región e impulsar el desarrollo "aguas abajo". Falta de coordinación y articulación de los actores del Sistema de Ciencia, Tecnología e Innovación tanto regionales como nacionales y el alto nivel de dependencia tecnológica, la cual se pone de manifiesto en el bajo número de investigadores, la poca producción de tecnología nacional y un elevado número de procesos industriales dependientes de tecnologías foráneas.

El presente proyecto se planteó como objetivo diseñar un Modelo de Política Pública de I+D+i para liderar, coordinar y 
direccionar el SRI del estado Bolívar, para el desarrollo de la capacidad de innovación en el sector de las PYMIS.

\section{Marco conceptual de la Investigación, Desarrollo Tecnológico e Innovación}

La Asociación Española de Normalización y Certificación (AENOR, 2007) publica la Norma de Gestión de la I+D+i, con el objeto de promover y sistematizar las actividades de Investigación, Desarrollo Tecnológico e Innovación en las industrias, define las actividades de innovación como la incorporación de tecnologías materiales e inmateriales, diseño industrial, equipamiento e ingeniería industrial, lanzamiento de la fabricación, comercialización de nuevos productos y procesos.

Hidalgo et al (2002) define las Políticas Públicas de I+D+i como el esfuerzo deliberado de influenciar la dirección y la tasa de desarrollo científico, tecnológico, de difusión y absorción de tecnologías avanzadas en la sociedad, por medio de la aplicación de recursos financieros, regulatorios y legislativos, dispositivos administrativos, educación y formación, en tanto que todos ellos estén afectados por la autoridad pública.

En la elaboración del glosario de términos de ciencia, tecnología e innovación productivas utilizados en América Latina y el Caribe, Lemarchand (20I0) define la Política de Ciencia y Tecnología como el conjunto de disposiciones y el ordenamiento jurídico que el estado debe adoptar para fomentar la investigación científica y tecnológica.

Durante los últimos 20 años, el interés de estudiar el éxito que ha alcanzado un determinado país asociado a la capacidad de innovación y las tecnologías en su sector industrial, se encuentra determinando a la estructura y funcionamiento del sistema de innovación a la cual pertenece.

Freeman (1987), Lundvall (1992) y Nelson (1993) destacan entre los primeros autores, que introducen el término de Sistema de Innovación, tomando interés a escala internacional el estudio y aplicabilidad para la creación de un entorno favorable que estimule el desarrollo de la I+D+i en las industrias. Específicamente cada autor realiza la siguiente definición de SI:

Freeman (1987) introduce el término Sistema Nacional de Innovación. Aparece, por primera vez en 1987, en una publicación sobre la innovación en Japón. Plantea que en los SNI se establece una red de instituciones en los sectores público y privados que interactúan para el desarrollo de nuevas tecnologías. Lundvall (1992) los elementos y las relaciones que interactúan en la producción, la difusión y el uso de nuevos conocimientos económicamente útiles y Nelson (1993) un conjunto de instituciones cuyas interacciones condicionan el desempeño innovador.

En el marco de un sistema de Innovación el manual de OSLO-OCDE (2006) se determinan los principales elementos constitutivos del medio en el cual opera la empresa, estos elementos son los siguientes: el sistema universitario, el sistema de información técnica especializada, la base científica y de investigación, los elementos comunes del conocimiento catalogado, las políticas de innovación y otras políticas gubernamentales que influyen en la innovación de la empresa, el marco legislativo y macroeconómico, la infraestructura y comunicaciones, incluyendo la red vial, las telecomunicaciones, las instituciones financieras que determinan por ejemplo la facilidad de acceso al capital de riesgo, la accesibilidad al mercado, la estructura industrial y el entorno competitivo, incluyendo la existencia de empresas suministradora.

El Sistema de Innovación se puede definir como el conjunto de instituciones y actividades relacionadas que interactúan con unidad de propósito, para la financiación, generación, difusión, transferencia y aplicación de conocimientos de I+D+i, dirigidas a satisfacer las necesidades y oportunidades de innovación en el mercado.

La Política Pública de I+D+i desde un enfoque de Sistema de Innovación se puede definir como el conjunto de actividades, procedimientos y acciones gubernamentales, para coordinar y direccionar el desarrollo de las actividades de ciencia, tecnología e innovación en las industrias, orientado al logro de nuevos productos o procesos o mejora de los mismos, que responda a las demandas de innovación en el mercado. Por lo tanto, las Política Pública de I+D+i deben impactar favorablemente en el crecimiento económico y social de un país o región, a través de la definición de aspectos legales, planes e instrumentos concertados y participativos con los diferentes actores del sistema de innovación, que intervienen en los programas de interés públicos de I+D+i.

\section{Metodología}

El proyecto representa una investigación no experimental de tipo proyectiva. Este tipo de investigación propone soluciones a una situación determinada a partir de un proceso de indagación, implica explorar, describir, explicar y proponer alternativas de cambios, mas no necesariamente ejecutar la propuesta Hurtado (2008).

El estudio proyectivo se divide en dos partes, primero se diagnóstica y se evalúa la situación actual con relación a la vinculación de las PYMIS con los demás miembros del SRI del estado Bolívar, a través del diseño y aplicación de tres tipos de encuestas, dirigido a tres miembros claves del SRI

ISSN: 07 I8-2724. (http://www.jotmi.org)

Journal of Technology Management \& Innovation (c) Universidad Alberto Hurtado, Facultad de Economía y Negocios. 
(PYMIS, centros y laboratorios de I+D de las universidades regionales, las instituciones financieras publica y privadas). En cada una de las encuestas se consideró el rol y las relaciones con la institución gubernamental que diseña y define la Política Pública de I+D+i.

En el estudio de campo, la muestra seleccionada para la aplicación de las encuestas y recolección de la información, represento un $47 \%$ de las PYMIS que realizan actividades de $\mathrm{I}+\mathrm{D}+\mathrm{i}$, este grupo de PYMIS, son reconocidas por los gremios y asociaciones empresariales e instituciones públicas y privadas de la región por poseer experiencia y realizar actividades de innovación.

La segunda encuesta se aplicó a los centros de I+D y laboratorios pertenecientes a las universidades regionales UNEXPO y UNEG representando el $100 \%$ de la población con respecto a los centros disponibles para apoyar las actividades de investigación de las PYMIS de la región, en las áreas de materiales y mecánica.

La tercera encuesta fué aplicada al sector financiero, la muestra estudiada fue el $27 \%$ de las 26 entidades financieras públicas y privadas ubicadas en la región, con el propósito de describir el tipo de instrumento financiero disponibles para las actividades de I+D+i que realizan las PYMIS.

En la segunda parte se diseña y se propone un modelo de política pública de I+D+i para liderar y coordinar las relaciones y vinculaciones de los actores que conforman el SRI en apoyo a la mejora de las capacidades de la I+D+i en las PYMIS.

\section{Resultados}

Con el análisis y procesamiento de los resultados como productos de la aplicación de las encuestas de investigación, se logró comprender las dificultades presentes en la vinculación actual de las PYMIS con los demás miembros del Sistema Regional de Innovación del estado Bolívar. Con esta información se diseño un modelo de Política Pública en I+D+i, para liderar, coordinar y dinamizar el SRI en apoyo a las PYMIS, con el propósito de mejorar su capacidad de innovación, que permita dar respuesta a las necesidades y oportunidades de innovación del mercado.

\section{Dificultades presentes en la vinculación actual de las PYMIS con los demás miembros del SRI del estado Bolívar}

I) Muy pocas PYMIS poseen formalmente un departamento de I+D y presentan escasa inversión en investigación y desarrollo.

2) Falta de personal especializado en las PYMIS para cumplir las funciones científica y tecnológica.

3) Las innovaciones de productos no cuentan con mecanismos de protección industrial, representando un riesgo de plagio las innovaciones generadas por las PYMIS.

4) Se detecta que la mayoría de las PYMIS consideran los instrumentos de políticas gubernamentales en $I+D+i$ no son suficiente, y los procedimientos administrativos no facilitan los procesos de innovación en la empresas.

5) El número de PYMIS beneficiadas por el financiamiento de instituciones públicas y privadas en $\mathrm{CTI}$ es casi inexistente.

6) No se cuenta con mecanismo de capital de riesgo para apoyar el procesos de I+D+i.

7) Falta de apoyo gubernamental en las compras del estado en materia de sustitución de importaciones.

8) En opinión de las empresas, la política de I+D+i en Venezuela se encuentra muy bien definidas, pero en su aplicación presenta pocos beneficios para la promoción y desarrollo de la I+D+i en las PYMIS de la región.

9) Las vinculación y la relación de las PYMIS con los centros de I+D de las universidades, se encuentran en niveles muy bajo para el trabajo de investigación conjunto. Ninguna de las empresas manifestó haber establecido alguna negociación de transferencia de tecnología con centros de I+D de la región.

10) Los niveles de cooperación empresarial para desarrollar actividades I+D+i, también representa una debilidad del sistema.

II) Limitaciones para el acceso a la información del mercado, con respecto a las necesidades y especificaciones técnicas de los productos susceptibles de sustitución de importaciones de las empresas de la zona.

12) La incidencia de la situación política, económica y social de país, afectan los procesos de inversión para las actividades de la l+D+i, por la incertidumbre que genera en las PYMIS.

\section{Fundamentos Básicos del Modelo de Política Pública de I+D+I}

Cuando los gobiernos centrales diseñan políticas públicas de I+D+i, lo hacen con el fin de impulsar la cultura de la ciencia, la tecnología y la innovación en la sociedad, mejorando en la mayoría de los casos la infraestructura científica y tecnológica de los centros y laboratorios de investigación, y la formación de investigadores de las universidades, donde el

ISSN: 07I 8-2724. (http://www.jotmi.org) 
sector de las PYMIS es poco beneficiadas de la transferencias de conocimientos de las universidades, del financiamiento público y privado, y de los instrumentos de política para las mejoras de sus capacidades de innovación.

En una economía global y de alta exigencia de mercados cada vez más competitivos se debe reorientar la política pública de $1+D+i$, no solo en establecer programas de financiamientos para los proyectos de $\mathrm{I}+\mathrm{D}+\mathrm{i}$ en las PYMIS sin promover la vinculación con sector científico y tecnológico de la universidades y centros de I+D. Sino en diseñar políticas integrales para promover una cultura de cooperación para la innovación industrial, partiendo de los planes y de los aspectos legales de la CTI, de las oportunidades de nuevos productos que motorizan las compras públicas en el mercado, del conocimiento de las necesidades y limitaciones de las capacidades de la I+D+i en las PYMIS para responder a la demanda del mercado, de las fortalezas y debilidades de la relación de las PYMIS con los demás actores del sistema de investigaciones a nivel internacional han planteado la necesidad de diseñar Políticas Públicas de $\mathrm{I}+\mathrm{D}+\mathrm{i}$, para que las empresas aumenten significativamente su inversión en investigación y desarrollo, sus esfuerzos de innovación, y realicen las actividades de I+D+i, a través de la articulación e integración y cooperación con los actores del sistema de innovación:

MANUAL DE BOGOTÁ elaborado por Jaramillo et al (200I), CEPAL (2004), Genatios y La Fuente (2004), UNESCO (2009), Lemarchand (20 I0), Casanova et al (20I I) y Anllo et al (20l I). Caso de estudio en países latinoamericanos e lberoamericanos.

KITAGAWA (2007), Motohashi (2008), Motohashi and Yun (2007), Herstatt et al (2008). Caso de estudio en los países asiáticos (Japón, China y la India), respectivamente.

COMISIÓN EUROPEA (2008), (20I0a), (20l0b) y (20I I), Fundación COTEC en España (2007), (2010) y (201I). Caso de estudios Países de la Unión Europea.

OECD (2007), (2008), (20I0a), (20I0b) y (20II) Caso de análisis y recomendaciones en más de 30 países de las regiones de América, Europa y Asia.

La propuesta del diseño del modelo de política pública de I+D+i para el desarrollo de las PYMIS, en su fase inicial se encuentra relacionadas a los modelos de proceso de innovación aportados por Kline y Rosenberg (1986), Rothwell, (1994), y AENOR (2007). Estos aportes se convierten en el conocimiento básico para entender la dinámica y las herramientas técnicas en el proceso interactivo de la innovación de productos, dentro de la estructura de la empresa. En todos estos modelos y teorías, se consideran las actividades de innovación como procesos interconectados, colocando en el inicio del estudio la etapa de la investigación de mercado que moviliza y organiza los intereses de la empresa para la innovación de nuevos productos o procesos. Lo que si deja claro los modelos y teorías de proceso de innovación, es que el mercado es la fuente de energía que mueve el sistema de gestión de la I+D+i de la empresa.

Con estos aportes se logró entender la evolución, la dinámica y las interacciones de procesos internos para que una empresa con capacidades propias en $I+D+i$, pueda iniciar $y$ culminar con éxito el desarrollo de un nuevo producto. Sin embargo, se tiene conocimiento y experiencia que la gran mayoría de las PYMIS, requiere del soporte de un Sistema de Innovación como una estructura de apoyo para emprender y desarrollar proyecto de $\mathrm{I}+\mathrm{D}+\mathrm{i}$, que genere productos para satisfacer las necesidades y las oportunidades de innovación en el mercado.

Como segunda fase, el diseño del Modelo de Políticas Públicas, se orienta a los estudios sobre los Sistemas de Innovación, que fueron iniciados por Freeman (1987), Lundvall (1992) y Nelson (1993) y de los Modelos de Sistema de Innovación que fueron presentados para comprender la estructura, la interacción y funcionamiento de la relación entre el gobierno, universidad, industrias y otras instituciones que conforman el Sl. Entre los diferentes modelos de SI se encuentran:

- $\quad$ El Triángulo de Sábato. Por Sábato, J y Botana, N (I 968).

- $\quad$ El Modelo de la Triple Hélice. Por Etzkowitz, H y Leydesdorff, L (2000).

- $\quad$ El Modelo Fundación COTEC (1998), en el Sistema Español de Innovación.

- El Modelo sobre el Sistema Español de Innovación, de Fernández de Lucio I, y Conesa F. (1996).

- El Marco para la Medición de la Innovación en la empresa, Manual de OSLO (2006).

La figura I, indica el modelo de la Política Publica de I+D+i para liderar y coordinar el Sistema Regional de Innovación en apoyo a las PYMIS, orientado al fortalecimiento de la capacidad de innovación.

\section{Descripción del Modelo de Política Pública de I+D+i, para Liderar y Coordinar el Sistema Regional de Innovación en apoyo a las PYMIS}

I. En esta primera etapa se debe tomar en cuenta para el diseño de política pública de $I+D+i$, para el desarrollo regional, revisar las principales fuentes de información de lineamientos gubernamentales en Ciencia, Tecnología, Innovación e Industrias, tales como: Plan de Desarrollo 
Económico y Social de la Nación, Política para el Desarrollo Industrial, Plan Nacional de CTI y los aspectos legales (Constitución, Leyes y Decretos), que orientan las actividades para el desarrollo de la I+D+i aplicado a la industria. Es de resaltar la importancia de lograr la unificación, coherencia y compromiso de los organismos de la administración pública, en el cumplimiento del marco legal y los objetivos definidos en los planes de CTI.

2. En la segunda etapa la institución gubernamental identifica y promueve las oportunidades de desarrollo de nuevos productos a través de la demanda en el mercado, creando un entorno favorable para la innovación. Es decir, el mercado se convierte en una fuente de información fundamental para el diseño de política pública de I+D+i, que moviliza la energía y visión de las PYMIS hacia la innovación.

3. La detección de las necesidades de las PYMIS y su nivel de capacidad en I+D+i para el desarrollo de nuevos productos es un asunto prioritario en el análisis y diseño de política pública de I+D+i por parte de la institución gubernamental, en poseer los conocimientos básicos de los factores internos y externos que dinamicen o bloqueen las actividades de innovación en la empresa.

4. En esta etapa la institución gubernamental responsable de diseñar la política pública de I+D+i para el desarrollo de las PYMIS en una región, parte del conocimiento de las tres primeras etapas, permitiendo identificar las oportunidades y los instrumentos que respondan a las necesidades de las PYMIS. Posteriormente se debe lograr poner en marcha y desplegar los lineamientos de políticas de I+D+i a los demás miembros del SRI, y su adecuada gestión y coordinación en el desarrollo de la capacidad de la innovación en las PYMIS.

5. En la quinta etapa la política pública de $++D+i$, juega un papel fundamental en la coordinación y articulación con

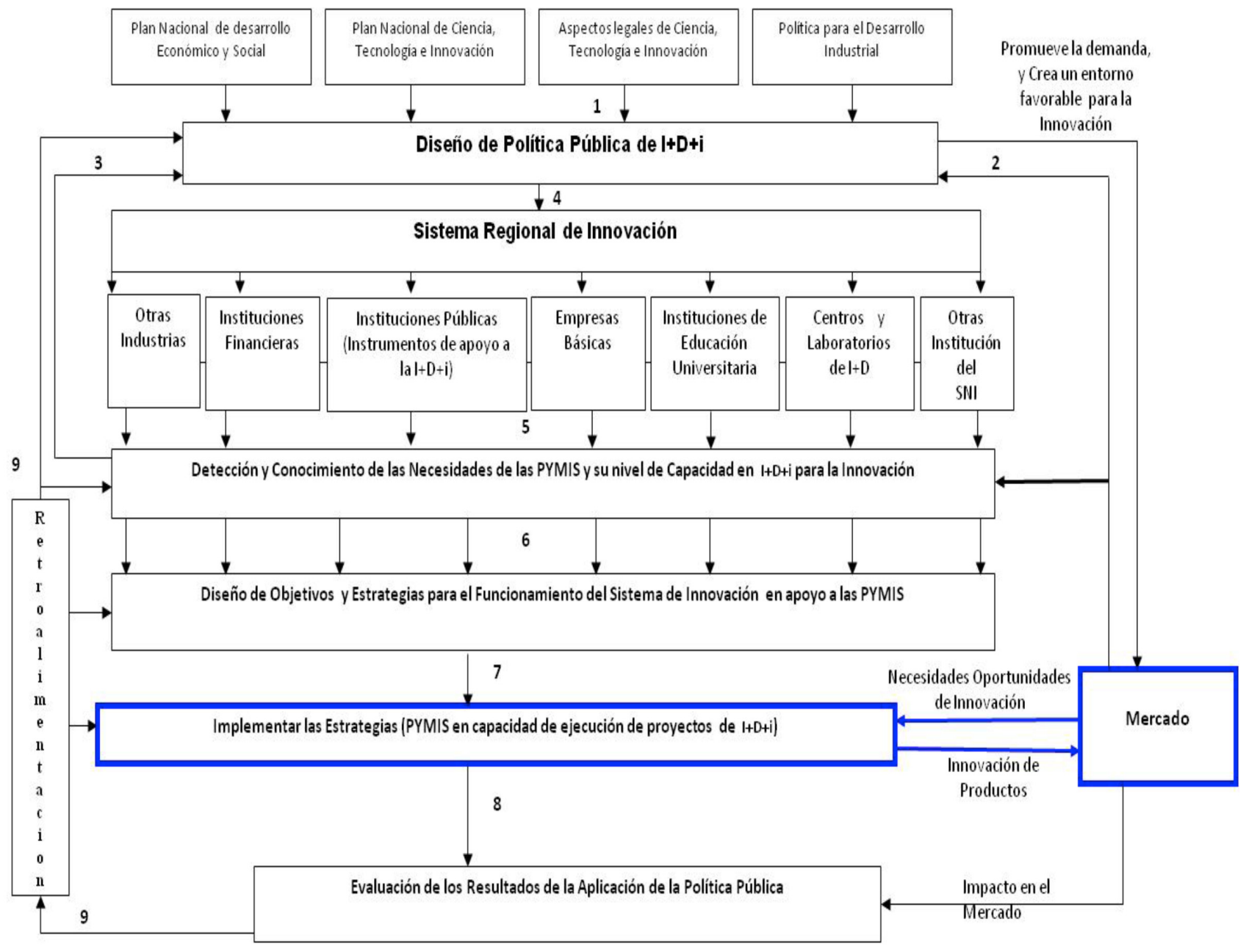

Figura I. Modelo de Política Pública de I+D+i para liderar y coordinar el SRI en apoyo a las PYMIS. Fuente: Elaboración propia

ISSN: 07 I8-2724. (http://www.jotmi.org)

Journal of Technology Management \& Innovation (C) Universidad Alberto Hurtado, Facultad de Economía y Negocios. 
los demás autores involucrados en el Sistema Regional de Innovación (Instituciones Públicas, Instituciones Financieras, Empresas Básicas Siderúrgicas y del Aluminio, Instituciones de Educación Universitaria, Centros y Laboratorios de Investigación y Desarrollo, Otras Industrias y los enlaces necesarios con instituciones del Sistema Nacional de Innovación). Además en el suministro de la información con respecto a la situación actual de las necesidades de la PYMI y su nivel de capacidad en I+D+i. Con estos requerimientos se tendrá en el SRI un diagnóstico del tipo de apoyo que demanda la PYMI, y que miembro del sistema podrá atender las necesidades detectadas.

6. Con los lineamientos generales de política pública de $I+D+i, y$ con el conocimiento de las necesidades de las PYMIS en I+D+i, por parte de todos los actores del SRI, se pasa en mejores condiciones a la etapa del diseño de objetivos y estrategias de Políticas de I+D+i en forma colectiva, que permita una mejor comunicación y el compromiso institucional para el optimo funcionamiento del SRI en apoyo a las PYMIS. En esta etapa se debe definir y acordar el rol que deben desempeñar los miembros del sistema para la ejecución de las estrategias, que contribuyan en el logro de los objetivos propuestos.

El diseño de las estrategias para el desarrollo de la innovación en las PYMIS, requiere una considerable liderazgo y coordinación por parte de la institución gubernamental responsable en lograr la articulación de los miembros del SRI, no solo en generar estrategias, sino también en la ejecución de las acciones acordadas, orientadas a elevar el nivel de capacidad de las PYMIS en I+D+i para el desarrollo de nuevos productos, respondiendo a las oportunidades de innovación en el mercado.

La aplicación de los pasos de la metodología $5 \mathrm{WIH}$, se adapta en el diseño de objetivos y estrategias de Políticas de I+D+i para el funcionamiento del SRI en apoyo a las PYMIS, facilitará la coordinación en las etapas de ejecución y de evaluación, ya que permite definir: QUÉ será hecho ("WHAT" ),QUIÉN deberá hacerlo ("WHO" ), CUÁNDO será hecho ("WHEN"), DÓNDE será hecho ("WHERE" ), PORQUÉ será hecho ("WHY"), CÓMO será hecho ("HOW").

7. Representa la etapa de implementación de las estrategias para la mejora de la capacidad de $I+D+i$ en las PYMIS. Permitirá que la PYMI, responda en mejores condiciones a las necesidades y oportunidades de innovación que presenta el mercado. De acuerdo con las responsabilidades asignadas a cada miembro del sistema, deben participar en la ejecución y acompañamiento de la estrategia acordada, bajo la coordinación de la institución gubernamental como dinamizador del SRI.
8. En esta etapa representa la evaluación de los resultados de la aplicación de la política pública de I+D+i, al comparar los resultados obtenidos con los acordados en la etapa 6, permitiendo verificar si las estrategias definidas con los autores del Sistema de Regional de Innovación lograron generar mejores indicadores de I+D+i en las PYMIS y en la región. Si los resultados son positivos las estrategias fueron bien diseñadas y ejecutadas. Por el contrario, si los resultados obtenidos por la ejecución de las estrategias no lograron generar en la PYMI una mejor capacidad de innovación, se debe revisar que todas las actividades fueron implementadas según lo acordado, con los autores del SRI.

La continuidad de los resultados indeseables, después de la etapa de ejecución, puede significar que las estrategias adoptadas no eran las más adecuadas o fueron mal implementadas.

Para un óptimo seguimiento y control de la gestión realizada por la institución gubernamental y los miembros del sistema, en el diseño y aplicación de política pública de I+D+i, es necesario la medición a través de indicadores de $I+D+i$, con el propósito de evaluar el impacto generado por las acciones en el SRI en apoyo al fortalecimiento de la capacidad de la $++D+i$ en las PYMIS.

9. La etapa de la retroalimentación y reflexión permite el aprendizaje y la mejora para el modelo propuesto de política pública de I+D+i. Saber qué tipo de decisión se debe tomar para la obtención de mejores resultados, si la política marcha en buena dirección o si es necesaria la aplicación de acciones correctivas en algunas de las etapas del modelo, que oriente la política pública de $1+D+i$ en el desarrollo industrial de la región. Además permite evaluar que instrumentos se adapta mejor a las PYMIS y cuales deben ser diseñado y coordinado con los autores del SRI.

\section{Conclusiones}

Las PYMIS del estado Bolívar cuentan con las siguientes condiciones favorables que deben ser aprovechadas para su desarrollo: marco legal y políticas públicas para apoyar y financiar las actividades de I+D+i. Centros y laboratorios de I+D de las universidades regionales con infraestructura científica y tecnológica al alcance de las PYMIS.Y el interés en la conformación de un SRI, para impulsar la vinculación y las relaciones de los miembros del sistema, en fortalecer el proceso de innovación en las PYMIS.

Del análisis de los resultados de cada uno de los miembros del SRI (PYMIS, centros y laboratorios de I+D de las universidades regionales, instituciones financieras públicas y privadas, e instituciones gubernamentales), se detecta la desarticulación del SRI del estado Bolívar, resultando indispensable 
una estructura de apoyo para el desarrollo de la $I+D+i$ en las PYMIS, como entono favorable para la innovación.

Reconocimiento de las PYMIS a nivel gubernamental al ser incorporados en los objetivos y estrategias en el plan de desarrollo económico y social de la nación, y en el plan nacional de CTI, con el propósito de mejorar sus actividades de I+D+i, y de facilitar las condiciones financieras públicas y privadas. Las Políticas Públicas en Venezuela se encuentran muy bien definidas, presentando debilidades y oportunidades de mejorar en el proceso de aplicación y evaluación. La política pública de I+D+i en Venezuela, presenta fallas estructurales, incidiendo en la baja capacidad de innovación en las PYMIS, representando una problemática por superar, lo relacionado a: la mejora en el nivel de inversión en $I+D+i$, consolidar la cooperación y las interrelaciones de los diferentes actores que conforman el sistema de innovación, un rol de liderazgo y coordinación de la institución gubernamental que diseña y ejecuta la políticas de I+D+i. Resultando una condición fundamental, que la política de innovación debe ser aplicada y evaluada para medir su aprendizaje $y$ cumplimiento.

El modelo plantea la relación entre la institución gubernamental y los miembros del SRI para promover y apoyar las actividades de $\mathrm{I}+\mathrm{D}+\mathrm{i}$ en las PYMIS. Resultando indispensable que la institución gubernamental responsable del diseño y la coordinación de la política pública de $\mathrm{I}+\mathrm{D}+\mathrm{i}$, tenga conocimiento de las necesidades, limitaciones y de las capacidades de la I+D+i en las PYMIS, para responder a la demanda del mercado. Así como también, conocer las fortalezas y debilidades de la relación de las PYMIS con los demás actores del SRI. Con estos conocimientos previos se podrá diseñar en mejores condiciones los instrumentos, objetivos y estrategias de política de $\mathrm{I}+\mathrm{D}+\mathrm{i}$, para coordinar, direccionar y evaluar el funcionamiento del SRI, enfocado al fortalecimiento de las capacidades de la I+D+i en las PYMIS para dar respuesta a las oportunidades de innovación de nuevos productos o procesos.

El desarrollo de las capacidades de la I+D+i en las PYMIS, depende del diseño y aplicación de política de publica de I+D $+\mathrm{i}$, como elemento dinamizador y articulador del SRI del estado Bolívar, orientado al fortalecimiento de las PYMIS, consolidándolas en organizaciones innovadoras que generen, aporten $o$ adapten los conocimientos científicos y tecnológicos, obteniendo nuevos productos o mejoras de procesos con un alto contenido de valor agregado regional. Mientras más disperso se encuentre el SRI en el estado Bolívar, la dependencia tecnológica seguirán guiando las relaciones comerciales en un mundo cada vez más globalizado. Se debe avanzar en la dirección de establecer alianzas entre los miembros del SRI, que promuevan la interdependencia de conocimientos en I+D+i, para afianzar el aprendizaje conjunto y el dominio tecnológico.

\section{Referencias}

AENOR (2007). Gestión de la I+D+i:Terminología y Definiciones, NORMAS UNE 166000:2006. AENOR. Madrid-España.

ANLLO, G., Lugones. G-, y Suarez, D. (20I I). Manual de Bogotá: hacia un formulario mínimo común. Estado de la Ciencia 2012. Red de Indicadores de Ciencia y Tecnología -lberoamericana e Interamericana- (RICYT).

http://www.ricyt.org/index.php?option=com_content\&view $=$ article\&id $=|49 \&|$ temid $=52$

ASAMBLEA Nacional Constituyente (1999). Constitución de la República Bolivariana de Venezuela. Gaceta Oficial $N^{\circ}$ $36.86030 / 12 / 1999$.

ASAMBLEA Nacional de la República Bolivariana de Venezuela (20I0). Ley Orgánica de Ciencia y Tecnología e Innovación, Gaceta Oficial No. 39.575, fecha 16/I2/2010. www. asambleanacional.gob.ve. Consultada el (I8/I2/20I0).

CASANOVA, L., Johnson, JD., Fonstand, NO., and Pietikäinen, A. (20II). Innovation in Latin America: Recent Insights (Chapter 2). The Global Innovation Index 20I I. Accelerating Growth and Development. INSEAD 20II.ISBN: 978-295222 10-I-6. Francia.

CEPAL (2004). Desarrollo Productivo en Economías Abiertas. La Comisión Económica para América Latina y el Caribe (CEPAL). Chile.

COMISIÓN EUROPEA. (1995). Libro Verde de Innovación. Bruselas, Bélgica.

COMISIÓN EUROPEA. (2010a) Europa 2020. Una Estrategia para un Crecimiento Inteligente, Sostenible e Integrador. Bruselas, Bélgica.

ETZKOWITZ, H., and Leydesdorff, L. (2000). The dynamics of innovation: from National Systems and "Mode 2" to a Triple Helix of university-industry-government relations. Research Policy 29_2000.109-123.

EUROPEAN COMMISSION (2008).A More Research-Intensive and Integrated European Research AreaScience, Technology and Competitiveness key figures report 2008/2009. Directorate-General for Research Communication Unit. Brussels, Bélgica.

EUROPEAN COMMISSION (20I0b). Europe 2020 Flagship Initiative Innovation Union. Brussels. 6.10.2010, COM (2010) 546 final. Bruselas, Bélgica.

ISSN: 07I 8-2724. (http://www.jotmi.org)

Journal of Technology Management \& Innovation (c) Universidad Alberto Hurtado, Facultad de Economía y Negocios. 
FERNÁNDEZ DE LUCIO, L., \& Conesa, F. (1996). Estructuras de Interfaz en el Sistema Español de Innovación. Su papel en la Difusión de Tecnologías. Centro de Transferencia de Tecnología. UPV, Universidad Politécnica de Valencia, España.

FREEMAN, C. (1987). Technology, Policy, and Economic Performance: Lessons from Japan. Pinter Publishers, London.

FUNDACIÓN COTEC (1998). Libro Blanco "El Sistema Español de Innovación Diagnóstico y Recomendaciones". España. http://www.cotec.es.

FUNDACIÓN COTEC. (2005). Libro Blanco de Innovación en el Principado de Asturias. Fundación Cotec para la Innovación Tecnológica. Madrid. España. http://www.cotec.es.

FUNDACIÓN COTEC (2007). Libro Blanco "Las Relaciones en el Sistema Español de Innovación. España. http://www. cotec.es.

FUNDACIÓN COTEC. (2010). Tecnología e Innovación en España. Informe Cotec 2010. Fundación Cotec para la Innovación Tecnológica. Madrid. España. http://www.cotec.es.

FUNDACIÓN COTEC. (20II). Tecnología e Innovación en España. Informe Cotec 20II. Fundación Cotec para la Innovación Tecnológica. Madrid. España. http://www.cotec.es.

FUNDACITE Bolívar (2008). Plan Regional de Ciencia, Tecnología e Innovación (2008-20I3). Estado Bolívar Venezuela. http://www.fundacite-bolivar.gob.ve/. Consultada (30/03/08).

GENATIOS, C., y La Fuente, M. (2004). Ciencia y Tecnología en América Latina. Ediciones OPSU.Venezuela.

GONZÁLEZ, J. (2008). Tesis Doctoral sobre un Modelo Sintético para el Diseño de Sistemas Regionales de Innovación en la Unión Europea. Universidad de Sevilla.

HERSTATT, C., Tiwari, R., y Buse, S. (2008). India's National Innovation System: Key Elements and Corporate Perspectives. Technology and Innovation Management- Hamburg University of Technology. Germany.

HIDALGO,A., Gonzalo, L., y Pavón, J. (2002). La Gestión de la Innovación y la Tecnología en las Organizaciones. Ediciones Pirámide, Madrid. España.

HURTADO, J. (2008). El Proyecto de Investigación. Ediciones Sypal Quirón.Venezuela.
JARAMILLO, H., Lugones, G., y Salazar, M. (200I). Manual de Bogotá - Normalización de Indicadores de Innovación Tecnológica en América Latina. Red Iberoamericana de Indicadores de Ciencia y Tecnología (RICYT). Colombia. http:// www.eclac.cl.

KITAGAWA, F. (2007). The Regionalization of Science and Innovation Governance in Japan. Regional Studies. Cambridge: Nov 2007. Tomo 4I, N 8; Pg. 1099.

KLINE, S., y Rosenberg, N. (1986). An overview of innovation, en The Positive Sum Strategy: Harnessing Technology for Economic Growth. Editado por Landau, R. y Rosenberg, N.,Washington.

LEMARCHAND, G. (20I0). Sistemas de Ciencia, Tecnología e Innovación en América Latina y el Caribe. Estudios y Documentos de Política Científica en ALC,Vol. I. UNESCO. Oficina Regional de Ciencia para América Latina y el Caribe. Uruguay.

Ley para la Promoción y Desarrollo de la Pequeña y Mediana Industria y Demás Unidades de Producción Social (2008). Decreto $N^{\circ}$ 6.215. Gaceta Oficial $N^{\circ}$ 5.890. Consultada (29/0I/09). www.inapymi.gob.ve/documentos/5890E\%20Supresion\%20FONCREI.pdf

LUNDVALL, B. (1992). National Systems of Innovation: Towards a Theory of Innovation and Interactive Learning, Pinter Publishers, London.

MANUAL DE OSLO -OCDE (2006). Guía para la Recogida e Interpretación de Datos Sobre Innovación. Tercera Edición. OECD- EUROSTAT. ISBN 84-6I I-278I-I -TRAGSA 2006. Comisión Europea.

MINISTERIO de Ciencia y Tecnología (2005). Plan Nacional de Ciencia, Tecnología e Innovación (2005-2030). www. gobiernoenlinea.ve/pncti.pdf. Venezuela. Consultada el (I5/I I/2005).

MINISTERIO de Planificación (2007). Plan de Desarrollo Económico y Social de la Nación 2007-200 I3. Venezuela. http://www.mpppd.gob.ve.

MOTOHASHI, K., and Yun, X. (2007). China's Innovation System Reform and Growing Industry and Science Linkages. Research Policy 36. 125I-1260.

MOTOHASHI, K. (2008). Growing R\&D Collaboration of Japanese Firms and Policy Implications for Reforming the National Innovation System. Asia Pacific Business Review. London: Jul 2008. Tomo I4, N 3; pg. 339. 
NELSON, R. (1993). National Innovation Systems. Oxford Up, Oford.

OECD. (2007). Innovation and Growth. Rationale for an Innovation Strategy.

OECD. (2008). Science, Technology and Industry Outlook 2008:Highlights.

OECD. (20/0a). Innovation Strategy. Ministerial report on the OECD Innovation Strategy. Innovation to strengthen growth and address global and social challenges. Key Findings. OECD Publishing. Paris.

OECD. (2010b). The OECD Innovation Strategy: Getting a Head Start on Tomorrow ISBN. 978-92-64-084704. OECD Publishing. Paris.

OECD. (20II). Demand-Side Innovation Policies. OECD Publishing. Paris.

PIÑERO, A., Rodríguez-Monroy, C., Arzola, M. (2008). Sistema Regional de Innovación para el Fortalecimiento de la I+D+i, en el Sector Industrial del estado Bolívar, Venezuela. Book of Abstracts - Iternational Conference on Industrial Engineering and Industrial Management. CIO 2008. ISBN 978-84-96394-86-5. Burgos-España.

ROTHWELI, R. (1994). Towards the fifth-generation innovation process, International Marketing Review, vol. II, $\mathrm{n}^{\circ} \mathrm{I}$. pp. 7-31.

SÁBATO, J., \& Botana, N. (1968). La Ciencia y la Tecnología en el Desarrollo Futuro de América Latina. Revista de la Integración n³, Noviembre 1968, Buenos Aires, Argentina.

UNESCO (2009). Declaración de América Latina y el Caribe en el decimo aniversario de la Conferencia Mundial Sobre la Ciencia, reunidos en Budapest (Hungría). www.unesco.org. uy/politicacientifica/budapest+10. Argentina. 